\title{
Iron - a key nexus in the virulence of Aspergillus fumigatus
}

\author{
Hubertus Haas* \\ Division of Molecular Biology/Biocenter, Innsbruck Medical University, Innsbruck, Austria
}

\section{Edited by:}

Reinhard Guthke, Hans Knöll

Institute, Germany

\section{Reviewed by:}

Sven Krappmann, Research Center for Infectious Diseases, Germany

Sean Doyle, National University of Ireland Maynooth, Ireland

*Correspondence:

Hubertus Haas, Division of Molecular Biology/Biocenter, Innsbruck Medical University, Fritz-Pregl-Str. 3, A-6020 Innsbruck, Austria.

e-mail: hubertus.haas@i-med.ac.at
Iron is an essential but, in excess, toxic nutrient. Therefore, fungi evolved fine-tuned mechanisms for uptake and storage of iron, such as the production of siderophores (low-molecular mass iron-specific chelators). In Aspergillus fumigatus, iron starvation causes extensive transcriptional remodeling involving two central transcription factors, which are interconnected in a negative transcriptional feed-back loop: the GATA-factor SreA and the bZip-factor HapX. During iron sufficiency, SreA represses iron uptake, including reductive iron assimilation and siderophore-mediated iron uptake, to avoid toxic effects. During iron starvation, HapX represses iron-consuming pathways, including heme biosynthesis and respiration, to spare iron and activates synthesis of ribotoxin AspF1 and siderophores, the latter partly by ensuring supply of the precursor, ornithine. In accordance with the expression pattern and mode of action, detrimental effects of inactivation of SreA and HapX are confined to growth during iron sufficiency and iron starvation, respectively. Deficiency in HapX, but not SreA, attenuates virulence of $A$. fumigatus in a murine model of aspergillosis, which underlines the crucial role of adaptation to iron limitation in virulence. Consistently, production of both extra and intracellular siderophores is crucial for virulence of $A$. fumigatus. Recently, the sterol regulatory element binding protein SrbA was found to be essential for adaptation to iron starvation, thereby linking regulation of iron metabolism, ergosterol biosynthesis, azole drug resistance, and hypoxia adaptation.

Keywords: iron, virulence, fungi, siderophore, isoprenoid, ergosterol, mevalonate, ornithine

\section{INTRODUCTION}

Iron is an essential nutrient for all eukaryotes and nearly all prokaryotes (Kaplan and Kaplan, 2009). As mono or diiron center as well as incorporated into heme or iron-sulfur clusters, this metal is an indispensable cofactor for a variety of cellular processes including electron transport, amino acid metabolism, and biosynthesis of DNA and sterols. Nevertheless, iron excess has the potential to catalyze the formation of cell-damaging reactive oxygen species (Halliwell and Gutteridge, 1984). The complex intertwining of iron metabolism and oxidative stress is emphasized by the iron-dependence of detoxification of oxidative stress as, e.g., catalases and peroxidases require heme as cofactor. Despite its high abundance in the Earth's crust, the bioavailability of iron is low owing to its oxidation into sparingly soluble ferric $\left(\mathrm{Fe}^{3+}\right)$ hydroxides by atmospheric oxygen. To ensure iron supply but to avoid iron toxicity, all organisms evolved sophisticated mechanisms to balance acquisition, storage, and consumption of iron. The control over access to iron is one of the central battlefields during infection as pathogens have to "steal" the iron from the host. Moreover, the mammalian innate immune system restricts access to iron by pathogens via a variety of mechanisms (Ganz, 2009; Weinberg, 2009).

Abbreviations: CBC, CCAAT-binding complex; FC, ferricrocin; FsC, fusarinine C; RIA, reductive iron assimilation; SB, siderophore biosynthesis; SIT, siderophore-iron transporter; TAFC, triacetylfusarinine C; TF, transcription factor.
Aspergillus fumigatus is a ubiquitous saprophytic fungus, which has become the most common air-borne fungal pathogen of humans (Tekaia and Latge, 2005). Clinical manifestations range from allergic reactions to life-threatening invasive disease, termed aspergillosis, particularly in immuno-compromised patients. The identification and functional characterization of 24 genes that are involved in iron homeostasis in A. fumigatus and/or Aspergillus nidulans, respectively, revealed significant insights into iron metabolism and its regulation (Table 1). A. nidulans is a less virulent $A$. fumigatus relative and longstanding genetic model organism. Inactivation of 10 of the 19 A. fumigatus genes caused defects in virulence. All of the virulence-associated genes are transcriptionally upregulated during iron starvation and encode functions that are important for survival during iron starvation, which emphasizes the crucial role of adaptation to iron starvation in virulence. This review summarizes the current knowledge on iron homeostasis and its role in virulence in Aspergillus spp.

\section{IRON ACOUISITION}

As microorganisms are believed to lack mechanisms for iron excretion, control of iron uptake is considered the major iron homeostatic mechanism (Haas et al., 2008). In contrast to various bacterial and fungal pathogens (Ratledge and Dover, 2000; Almeida et al., 2009), both A. fumigatus and A. nidulans lack systems for direct uptake of host iron sources such as heme, ferritin, or transferrin (Eisendle et al., 2003; Schrettl et al., 2004a). 
Table 1 | Functionally analyzed proteins involved in iron homeostasis in A. fumigatus and $A$. nidulans.

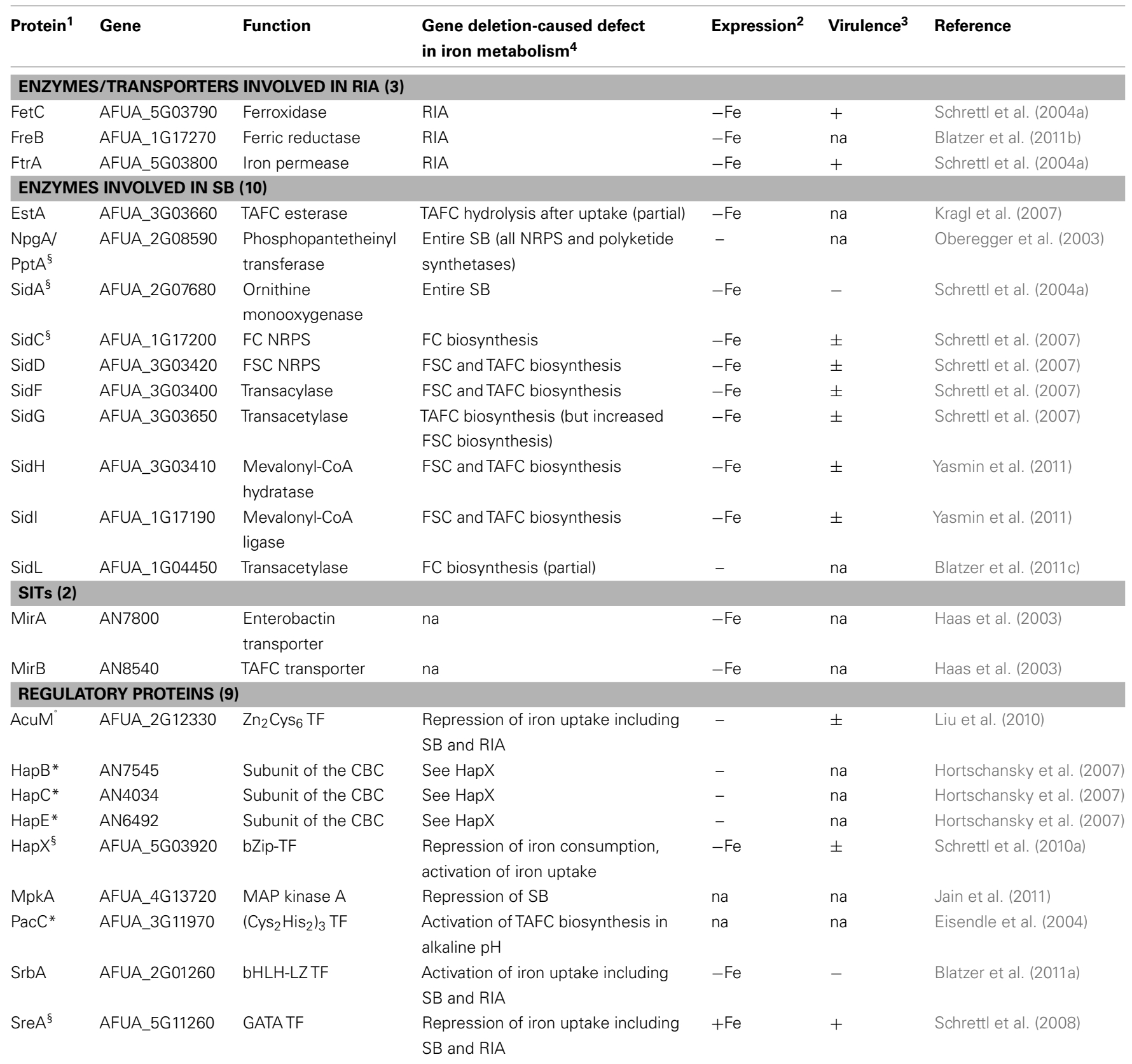

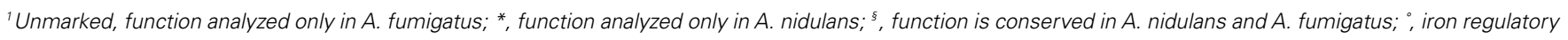
function found only in A. fumigatus but not in A. nidulans.

${ }^{2}-F e$, transcriptional upregulation during iron starvation; + Fe, transcriptional upregulation during iron sufficiency; - , constitutively expressed.

${ }^{3}+$, Virulent; \pm , partially attenuated virulent; - , avirulent.

$2,3,4$ na, not analyzed.

Both Aspergillus species employ low-affinity ferrous $\left(\mathrm{Fe}^{2+}\right)$ iron acquisition as well as siderophore-assisted iron uptake, a highaffinity ferric iron uptake system (Eisendle et al., 2003; Schrettl et al., 2004a). In contrast to A. nidulans, A. fumigatus possesses a second high-affinity iron uptake system, termed reductive iron assimilation (RIA). Schemes of the mechanisms for iron uptake and storage employed by Aspergillus spp. are found in recently published reviews (Haas et al., 2008; Schrettl and Haas, 2011).

\section{LOW-AFFINITY IRON UPTAKE}

At the molecular level, low-affinity iron uptake has been characterized exclusively in Saccharomyces cerevisiae. The relevant permeases are not specific for ferrous iron but additionally transport 
other metals, such as copper and zinc (Kaplan and Kaplan, 2009).

\section{REDUCTIVE IRON ASSIMILATION}

Reductive iron assimilation starts with reduction of ferric iron sources to the more soluble ferrous iron by plasma membranelocalized metalloreductases (Kosman, 2010). A. fumigatus encodes 15 putative metalloreductases indicating possible redundancy of this enzyme system. The metalloreductase FreB has recently been shown to be involved in RIA (Blatzer et al., 2011b). The reduced ferrous iron is re-oxidized and imported by a protein complex consisting of the ferroxidase FetC and the iron permease FtrA (Schrettl et al., 2004a).

\section{SIDEROPHORE-MEDIATED IRON UPTAKE}

The siderophores produced by A. nidulans and A. fumigatus are shown in Figure 1. Both fungal species excrete two siderophores, fusarinine $\mathrm{C}(\mathrm{FsC})$ and its derivative triacetylfusarinine $\mathrm{C}$ (TAFC), to mobilize extracellular iron. The ferri-forms of $\mathrm{FsC}$ and TAFC are taken up by siderophore-iron transporters (SIT), which constitute a subfamily of the major facilitator protein superfamily. SIT act most likely as proton symporters energized by the plasma membrane potential (Haas et al., 2003; Philpott and Protchenko, 2008). SIT-mediated iron uptake appears to be universally conserved in the fungal kingdom, even in species not producing siderophores such as S. cerevisiae, Candida spp., and Cryptococcus neoformans (Schrettl et al., 2004b; Haas et al., 2008; Jung and Kronstad, 2008; Philpott and Protchenko, 2008; Nevitt and Thiele, 2011). A likely reason is the dramatically increased solubility and therefore bioavailability of iron chelated by siderophores. Moreover, siderophores might play a role in microbial warfare as chelation of environmental iron by siderophore-types that are not recognized by competitors might be used to starve competitors of iron. This is counteracted by evolving transporters that recognize xenosiderophores, i.e., siderophores that are not produced by the organism, which enables "stealing" of siderophores. This scenario is supported by the fact that most siderophore-producing bacteria and fungi possess xenosiderophore-specific SITs (Haas et al., 2008). A. fumigatus and A. nidulans encode 10 and 7 putative
SITs, respectively (Haas et al., 2008). Heterologous expression in a S. cerevisiae mutant lacking high-affinity iron uptake indicated that the A. nidulans SITs MirA and MirB transport the bacterial siderophore enterobactin and TAFC, respectively (Haas et al., 2003).

After uptake, the intracellular release of iron from TAFC and $\mathrm{FsC}$ involves hydrolysis of the siderophore backbones by the esterase EstB (Kragl et al., 2007).

\section{IRON STORAGE}

Extracellular siderophores are employed by most bacterial and some plant species. In contrast, intracellular siderophores are only found in siderophore-producing fungi. A. fumigatus produces two different intracellular siderophores (Figure 1), ferricrocin (FC) for hyphal iron storage and distribution and its derivative hydroxyferricrocin (HFC) for conidial iron storage (Schrettl et al., 2007; Wallner et al., 2009). A. nidulans lacks HFC and employs FC for both hyphal and conidial iron storage (Eisendle et al., 2003). Additionally, the iron-inducible expression of CccA, the ortholog of the vacuolar iron importer Ccclp from S. cerevisiae (Kaplan and Kaplan, 2009), indicates vacuolar iron storage in A. fumigatus (Schrettl et al., 2008). In contrast to bacteria, plants, and animals, fungi lack ferritin-mediated iron storage and detoxification.

\section{SIDEROPHORE BIOSYNTHESIS}

Fusarinine C consists of three $N^{5}$-anhydromevalonyl- $N^{5}$ hydroxyornithine residues cyclically linked by ester bonds. TAFC is the $N^{2}$-acetylated FsC. FC is a cyclic hexapeptide with the structure Gly-Ser-Gly- $\left(N^{5} \text {-acetyl- } N^{5} \text {-hydroxyornithine }\right)_{3}$ and HFC is the hydroxylated FC (Haas et al., 2008). The siderophore biosynthesis (SB) pathway was characterized by reverse genetics and is shown in Figure 2. The first committed step in the biosynthesis of all four siderophores is the hydroxylation of ornithine catalyzed by the ornithine monooxygenase SidA (Eisendle et al., 2003; Schrettl et al., 2004a). Subsequently, the pathways for biosynthesis of extra and intracellular siderophores split. For extracellular SB the transacylase SidF transfers anhydromevalonyl to hydroxyornithine (Schrettl et al., 2007). The required anhydromevalonylCoA moiety is derived from mevalonate by CoA-ligation and

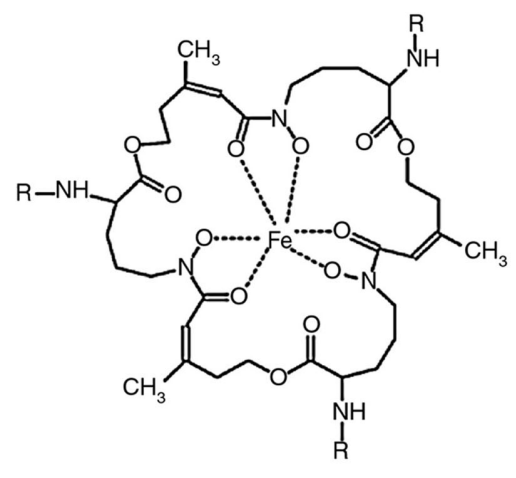

FsC/TAFC

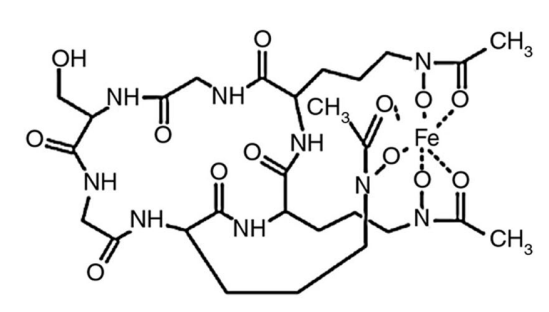

FC

FIGURE 1 | Siderophores produced by $A$. fumigatus and $A$. nidulans: $R=H$ in fusarinine $C(F s C)$ and $R=$ acetyl in triacetylfusarinine $C$ (TAFC); the hydroxylation site in hydroxyferricrocin (HFC) is unknown. The siderophores are shown in the ferric $\left(\mathrm{Fe}^{3+}\right)$ form. 


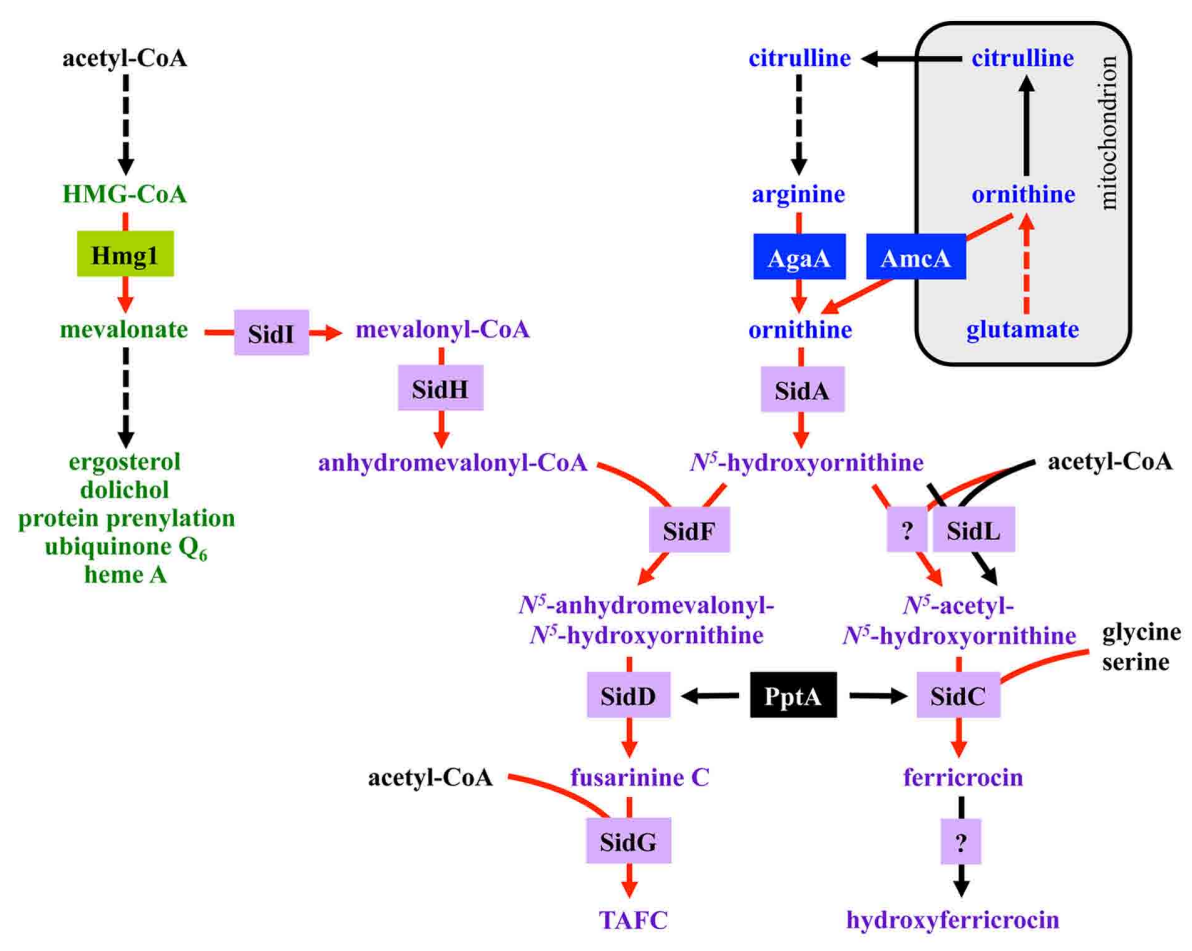

FIGURE 2 | Siderophore biosynthesis (in purple) and its links to the isoprenoid biosynthesis (in green) as well as ornithine/arginine metabolism (in blue). The enzymes, boxed in respective colors, are described in the text and Table 1. Enzymatic steps transcriptionally upregulated during iron starvation are marked by red arrows. Broken arrows denote reactions involving more than one enzyme. dehydration catalyzed by SidI and SidH, respectively (Yasmin et al., 2011). The acetylation of hydroxyornithine for intracellular SB involves two transacetylases, the constitutively expressed SidL and an unidentified enzyme, the activity of which is upregulated by iron starvation (Blatzer et al., 2011c). Assembly of FsC and FC is catalyzed by two different non-ribosomal peptide synthetases (NRPS), SidD and SidC, respectively. TAFC and HFC are formed by SidG-mediated $N^{2}$-acetylation of FsC and hydroxylation of FC, respectively (Eisendle et al., 2003; Schrettl et al., 2007). NRPS, polyketide synthases, and the lysine-biosynthetic $\alpha$-aminoadipate reductase depend on activation by the $4^{\prime}$-phosphopantetheinyl transferase. Consistently, the $4^{\prime}$-phosphopantetheinyl transferases NpgA and PptA are essential for SB in A. nidulans and A. fumigatus, respectively (Oberegger et al., 2003; Allen et al., 2011). Consequently, it is not surprising that this enzyme was found to be indispensable for phytopathogenic fungi (Horbach et al., 2009).

\section{GENOMIC ORGANIZATION OF GENES INVOLVED IN HIGH-AFFINITY IRON ACQUISITION}

Most of the described SB genes above are organized in three gene clusters containing additional genes encoding putative siderophore transporters, the TAFC esterase EstB and genes with uncharacterized functions (Schrettl et al., 2008, 2010a). Exceptions are the genes encoding SidA, SidL, and NpgA/PptA. Notably, SidL and NpgA/PptA are special for another reason as their expression is, in contrast to the other identified structural components of siderophore metabolism, not regulated by iron availability (Oberegger et al., 2003; Blatzer et al., 2011c). Moreover,
NpgA/PptA is not exclusively involved in SB. In contrast to $A$. fumigatus and A. nidulans, SidA ortholog-encoding genes are clustered with siderophore NRPS in various other fungi, e.g., Ustilago maydis and Neurospora crassa (Haas et al., 2008). The genes encoding FtrA and FetC form a gene cluster with a common promoter region.

\section{DEFECTS CAUSED BY SIDEROPHORE-DEFICIENCY}

Genetic elimination of extracellular siderophores $(\Delta$ sidF and $\Delta$ sidD mutants) decreases growth, conidiation, and oxidative stress resistance during iron limitation but not during iron sufficiency, which enables compensation by other iron acquisition systems (Schrettl et al., 2007). Elimination of intracellular siderophores ( $\Delta$ sidC mutant) reduces conidiation and blocks sexual development (as shown in A. nidulans) due to the role of FC in intracellular iron transport from substrate-contacting hyphae into aerial hyphae (Eisendle et al., 2006; Schrettl et al., 2007; Wallner et al., 2009). FC-deficiency decreases the conidial iron content by about $50 \%$, which impairs iron-dependent enzymes such as aconitase and catalase A, and thereby decreases conidial size and conidial resistance to oxidative stress (Schrettl et al., 2007; Wallner et al., 2009). Moreover, the lack of FC-mediated iron storage ( $\Delta$ sidC mutants) delays germination during iron starvation (Schrettl et al., 2007). Inactivation of the entire siderophore system ( $\Delta$ sidA mutant) combines the defects caused by inactivation of either extra or intracellular SB and renders A. fumigatus extremely sensitive to iron starvation (Schrettl et al., 2004a, 2007). 
Both extra and intracellular siderophores are crucial for virulence as elimination of the entire $\mathrm{SB}(\Delta \operatorname{sid} A$ mutant) results in absolute avirulence of $A$. fumigatus in a murine model of invasive pulmonary aspergillosis (Schrettl et al., 2004a; Hissen et al., 2005), while deficiency in either extracellular ( $\Delta$ sidI, $\Delta$ sidH, $\Delta$ sidF, or $\Delta$ sidD mutants) or intracellular siderophores ( $\Delta$ sidC mutants) causes partial attenuation of virulence (Schrettl et al., 2007; Yasmin et al., 2011). Conidial FC appears to play a particularly crucial role during initiation of infection because restoration of the conidial HFC content by supplementation with FC during conidiation partially cures the virulence defect of $\Delta$ sidA conidia (Schrettl et al., 2007). SidG-deficiency, which eliminates TAFC production with concomitant increase of FsC production, affects neither growth nor virulence, indicating that the structural differences between these two siderophores do not play a role in these settings (Schrettl et al., 2007). Consistent with a role in iron acquisition during infection, A. fumigatus' siderophores are able to remove iron from host sources, such as transferrin (Hissen et al., 2004; Hissen and Moore, 2005).

Blocking RIA ( $\triangle \mathrm{ftr} A$ mutant) does not affect virulence of $A$. fumigatus (Schrettl et al., 2004a). Nevertheless, a putative role of RIA in virulence is indicated by several lines of evidence: (i) elimination of extracellular siderophores causes only partial attenuation of virulence, (ii) mutants lacking both RIA and the siderophore system ( $\triangle$ ftrA $\Delta$ sidA double mutant) are unable to grow unless supplemented with siderophores or extremely high iron concentrations fueling low-affinity iron uptake (Schrettl et al., 2004a), and (iii) genome-wide expression profiling demonstrated induction of both the siderophore system and RIA during murine infection (McDonagh et al., 2008). Consistently, RIA has been shown to be crucial for virulence of the siderophore-lacking species C. albicans and C. neoformans (Ramanan and Wang, 2000; Jung et al., 2008).

The siderophore system is important not only for extra, but also for intracellular growth as defects in the siderophore system decrease intracellular growth and survival of A. fumigatus after phagocytosis by murine alveolar macrophages, which represent the first line of defense in the lung during pulmonary aspergillosis (Schrettl et al., 2010b). Furthermore, impairment of $\mathrm{SB}$ changes the immune response of macrophages after phagocytosis of A. fumigatus (Seifert et al., 2008). In agreement, the siderophore system is also critical for virulence of Histoplasma capsulatum, a dimorphic fungal pathogen replicating in the yeast form within macrophages (Hwang et al., 2008). The evolutionary conserved role of siderophores in virulence has been confirmed in various other aspergillosis infection models, i.e., a murine cutaneous model, Drosophila melanogaster, and Galleria mellonella (Ben-Ami et al., 2010; Chamilos et al., 2010; Slater et al., 2011). Moreover, SB is indispensable for the virulence of various phytopathogenic ascomycetes (Oide et al., 2006; Greenshields et al., 2007). In contrast, SB is dispensable and RIA is essential for phytopathogenicity of U. maydis (Mei et al., 1993; Eichhorn et al., 2006).

\section{INTEGRATION OF THE SIDEROPHORE-BIOSYNTHETIC PATHWAY IN THE GENERAL METABOLISM}

During iron starvation, siderophore production reaches up to $10 \%$ of the biomass. In addition, iron starvation dramatically remodels the free amino acid pool of A. fumigatus with eight amino acids increasing and three amino acids decreasing more than 1.5-fold (Schrettl et al., 2010a). Among these changes, the approximate sevenfold increase of the siderophore precursor ornithine during iron starvation compared to sufficiency indicates that the enormous ornithine demand for SB is matched by active upregulation of biosynthesis and not by de-repression via its consumption. Consistently, blocking siderophore-mediated ornithine consumption by inactivation of SidA ( $\Delta$ sidA mutant) causes a further 2.9-fold increase of the ornithine pool during iron starvation (Schrettl et al., 2010a).

Ornithine is produced in the mitochondrion, and its biosynthetic pathway as well as export via the transporter AmcA to the cytosol is transcriptionally upregulated under iron deprivation (Schrettl et al., 2010a; Figure 2). Moreover, ornithine is a precursor of arginine and the conversion of arginine to ornithine in the cytosol by the arginase AgaA is likewise upregulated in response to iron starvation. The metabolic commitment required for siderophore production must however be balanced against the need to maintain other cellular functions as arginine itself is vital for protein biosynthesis. Recently, arginine was found to allosterically activate SidA enzyme activity (Frederick et al., 2011), which appears to connect siderophore production to cellular amino acid homeostasis: SB is stimulated only if the cytosolic arginine pool is sufficient for all of the cell's needs.

Consistent with mevalonate being a precursor for TAFC biosynthesis (Figure 2), overexpression of the mevalonate-producing HMG-CoA reductase Hmgl increases TAFC production, while lovastatin-mediated Hmgl inhibition blocks TAFC biosynthesis in A. fumigatus (Yasmin et al., 2011). Mevalonate is an intermediate of the isoprenoid biosynthetic pathway with ergosterol as the major product, which is one major target of antifungal treatment, i.e., amphotericin $\mathrm{B}$ and azoles. In contrast to siderophore production, iron starvation decreases the cellular ergosterol level due to the iron-requirement of ergosterol biosynthesis (Yasmin et al., 2011). Concordant with bilateral demand for mevalonate, blocking mevalonate consumption for TAFC biosynthesis $(\Delta$ sidI mutant) alters the sterol composition and increases lovastatin resistance during iron starvation (Yasmin et al., 2011). These data demonstrate that statins such as lovastatin, which are widely used as cholesterol-decreasing drugs, have an additional target in siderophore-producing fungi. As SB is crucial for virulence statins might be useful to combat infections with siderophore-producing fungi.

\section{REGULATION OF IRON METABOLISM AND ITS ROLE IN VIRULENCE}

Iron starvation has been shown to cause extensive transcriptional remodeling in A. fumigatus with about $13 \%$ of the genes responding to iron availability (Schrettl et al., 2008, 2010a). As shown in Figure 3, in both A. fumigatus and A. nidulans maintenance of iron homeostasis involves the two central transcription factors (TF) SreA and HapX (Haas et al., 1999; Hortschansky et al., 2007; Schrettl et al., 2008, 2010a). During iron sufficiency, the GATA-factor SreA, postulated by bioinformatic analyses to recognize the consensus sequence ATCWGATAA, represses high-affinity iron uptake, including RIA and the siderophore system, to avoid 


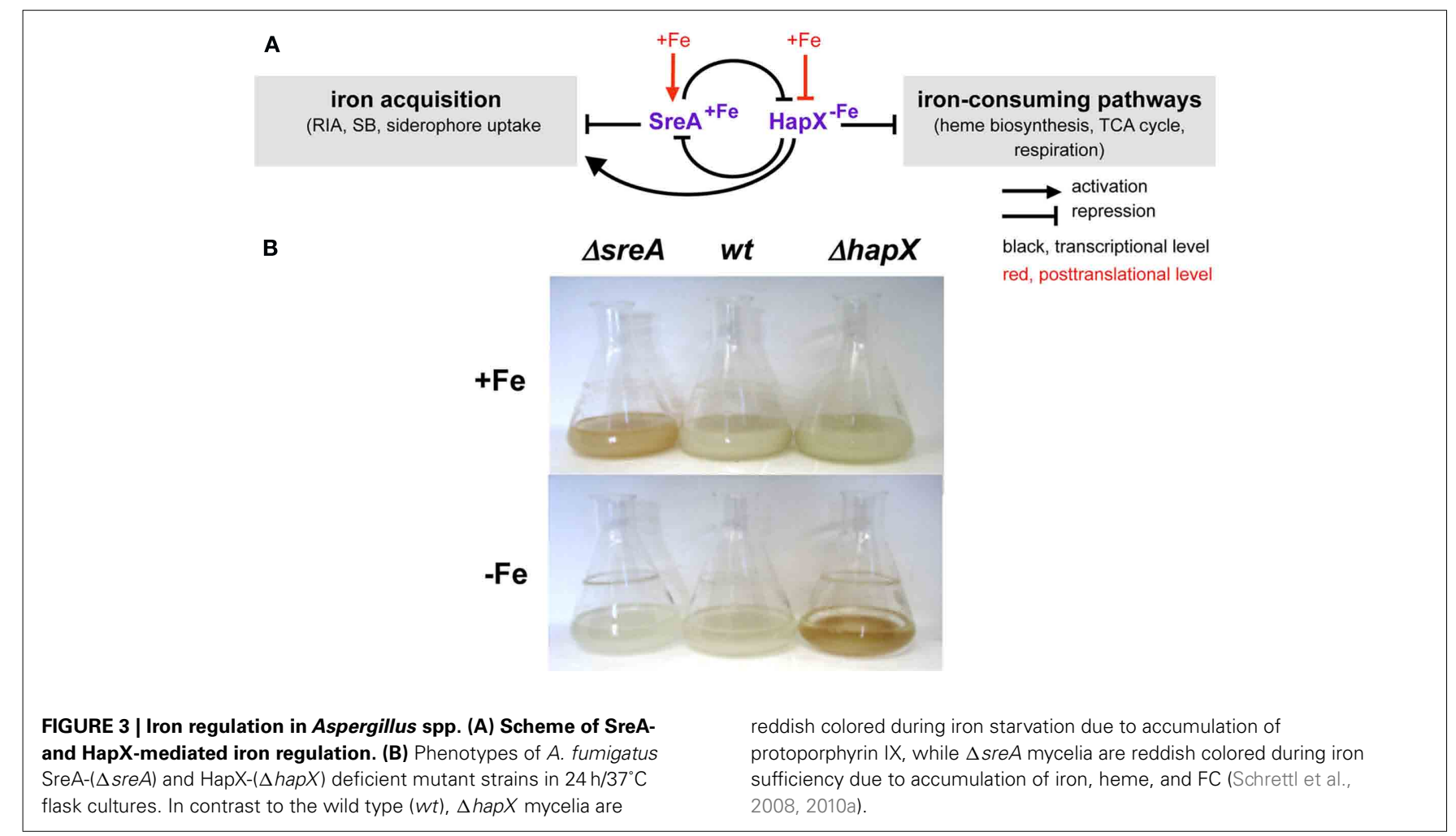

iron toxicity (Schrettl et al., 2008). During iron starvation, the bZip-TF HapX represses iron-consuming pathways such as heme biosynthesis, respiration, and ribosome biogenesis to spare iron (Oberegger et al., 2001, 2002; Schrettl et al., 2008, 2010a). Furthermore, HapX activates synthesis of the ribotoxin AspF1 and siderophores in A. fumigatus, the latter partly by coordinating SB with supply of its precursor ornithine (Schrettl et al., 2010a). The metabolic deregulation caused by deficiency in either SreA or HapX causes reddish hyphal pigmentation (Figure 3). SreA and HapX are interconnected in a negative feed-back loop: SreA represses expression of hapX during iron sufficiency, while HapX represses sreA during iron starvation. Additionally, both SreA and HapX appear to be regulated posttranslationally by iron blocking HapX function and activating SreA function (Haas et al., 1999; Hortschansky et al., 2007). In S. pombe, posttranslational iron sensing by the HapX and SreA orthologs involves the monothiol glutaredoxin Grx4 (Mercier and Labbe, 2009; Jbel et al., 2011; Kim et al., 2011). In A. nidulans and A. fumigatus, inactivation of both HapX and SreA is synthetically lethal underlining the critical role of iron homeostasis in cellular survival (Hortschansky et al., 2007; Schrettl et al., 2008, 2010a). In agreement with their expression pattern and mode of action, detrimental effects of inactivation of SreA or HapX are confined to growth during iron sufficiency or starvation, respectively (Figure 3). Deficiency in HapX, but not SreA, attenuates virulence of $A$. fumigatus in murine models of aspergillosis (Schrettl et al., 2008, 2010a), which emphasizes the crucial role of adaptation to iron limitation in virulence. Most fungal species possess orthologs to SreA and HapX and the important role of HapX orthologs in virulence has been demonstrated in $C$. albicans and C. neoformans (Labbe et al., 2007; Haas et al., 2008;
Jung et al., 2010; Hsu et al., 2011). Similar to A. fumigatus, the SreA ortholog Sful is dispensable for systemic virulence of C. albicans (Chen et al., 2011). However, Sful is crucial for persistence of this commensal in the iron-rich gut, which impressively illustrates the importance of adaptation to opposing conditions of iron availability for survival. Remarkably, the fungal prototype S. cerevisiae lacks orthologs of SreA, HapX, and SrbA and employs entirely different regulators, Aft1/2 and Cth1/2, which are conserved only in closely related Saccharomycotina species (Haas et al., 2008; Kaplan and Kaplan, 2009).

\section{INTERCONNECTION OF IRON METABOLISM WITH OTHER REGULATORY CIRCUITS: OXYGEN, REDOX PH, GLUCONEOGENESIS, MAP KINASE SIGNALING, ZINC}

Due to the central metabolic role of iron, a variety of regulatory circuits affect cellular iron handling. As obligate aerobic organisms, Aspergilli rely on respiration, which is highly iron-dependent. Therefore, oxygen supply largely influences iron metabolism and vice versa. Recently, proteomic analysis of A. fumigatus revealed that hypoxia increases the production of proteins involved in glycolysis, the TCA-cycle, and respiration, which is paralleled by increased cellular iron, heme, copper, and zinc contents (Vodisch et al., 2009). The increase in iron/heme is attributable to the iron/heme-dependence of the TCA-cycle and respiration. In $A$. fumigatus, hypoxic adaptation involves SrbA, a member of the "sterol regulatory element binding protein (SREBP)" TF family, which is conserved in most eukaryotes (Willger et al., 2008). These TFs are activated by cellular sterol-depletion to maintain sterol homeostasis (Bien and Espenshade, 2010). In A. fumigatus, SrbA-deficiency decreases the cellular ergosterol content 
and resistance against azole drugs and blocks hypoxic growth as well as virulence (Willger et al., 2008). Recently, SrbA was found to activate siderophore-mediated iron uptake in response to hypoxia and iron starvation in part by transcriptional activation of HapX (Blatzer et al., 2011a). In agreement with SrbA being involved in iron homeostasis, defects in hypoxic growth and azole resistance caused by SrbA-deficiency are at least partially cured by increased iron supplementation and in particular by de-repression of iron uptake via simultaneous inactivation of SreA (Blatzer et al., 2011a). SrbA is transcriptionally upregulated during hypoxia and iron starvation, in both cases likely in response to sterol-depletion and autoregulation as sterol biosynthesis depends on both oxygen and iron. During iron starvation, consumption of the sterol intermediate mevalonate by SB might play an additional role (see above). In agreement, the transcriptional activation of SrbA during iron starvation is independent of SreA and HapX. These data indicate that A. fumigatus senses iron not only via HapX and SreA but also via ergosterol biosynthesis and SrbA. Taken together, SrbA coordinates ergosterol biosynthesis and iron metabolism to mediate hypoxia responses and azole resistance. Growth defects during iron starvation of respective gene deletion mutants indicate similar functions in iron homeostasis of the SrbA orthologs in S. pombe and C. neoformans (Hughes et al., 2005; Chang et al., 2007). Moreover, the SrbA ortholog is also crucial for virulence in C. neoformans (Chun et al., 2007). Consequently, the virulence defect of SrbA mutants is possibly attributable not only to the defects in adaptation to hypoxia, but also iron starvation. S. cerevisiae lacks an SrbA ortholog and the TFs mediating hypoxic adaptation (Hap1, Mot1, Rox1) are not conserved in Aspergilli (Willger et al., 2008), which might be explained by $S$. cerevisiae being an facultative anaerobe.

As shown in A. nidulans, HapX functions via physical interaction with the DNA-binding CCAAT-binding complex (CBC; Hortschansky et al., 2007). The CBC is a heterotrimeric DNAbinding complex, which is conserved in all eukaryotes. In $A$. nidulans, inactivation of either one of its subunits, НapB, НapC, or HapE phenocopies HapX inactivation with respect to the defects in adaptation to iron starvation. However, the CBC has HapXindependent functions and is speculated to affect expression of about $30 \%$ of all genes. Consistently, CBC-deficiency results in decreased growth and sporulation during both iron sufficiency and starvation (Hortschansky et al., 2007). The mode of discrimination between HapX targets and the remaining CBC targets remains to be elucidated. As mentioned in the introduction, iron metabolism and oxidative stress are intimately intertwined. Therefore it is particularly interesting that, as shown in A. nidulans, the $\mathrm{CBC}$ senses the redox state of the cell via oxidation and thioredoxin-mediated reduction of evolutionary conserved thiol groups within the HapC histone fold motif (Thon et al., 2010). Oxidation blocks CBC formation and nuclear localization. In line with a role in redox regulation, CBC-deficiency impairs the oxidative stress response. The impact of iron availability on the oxidative stress detoxification system is indicated by iron starvation-mediated transcriptional downregulation of the heme-dependent hyphal catalase $\mathrm{B}(\mathrm{Cat} \mathrm{B})$ and upregulation by the Cu/Zn-superoxide dismutase (SodA; Oberegger et al., 2000, 2001).
Excessive iron uptake caused by SreA-deficiency transcriptionally upregulates both enzymes (Oberegger et al., 2001).

The ambient $\mathrm{pH}$ impacts iron availability as alkaline conditions decrease iron solubility. In line, neutral compared to acidic conditions upregulate SB and uptake in A. nidulans mediated by the pH-reactive TF PacC (Eisendle et al., 2004). Similarly, PacC orthologs mediate upregulation of high-affinity iron uptake - in these species RIA - during alkaline conditions in S. cerevisiae, C. albicans, and C. neoformans (Lamb et al., 2001; Baek et al., 2008). Virulence of C. albicans but not C. neoformans depends on its $\mathrm{PacC}$ ortholog, which is most likely attributable to the occupation of different host niches by these two pathogens (Nobile et al., 2008; O'Meara et al., 2010).

Interestingly, deficiency in the TF AcuM, which is required for gluconeogenesis, decreases siderophore production in A. fumigatus, but not in A. nidulans, and attenuates virulence in A. fumigatus (Liu et al., 2010). Though it is unclear if its effects are direct, AcuM appears to transcriptionally repress SreA.

Recently, iron starvation was found to trigger phosphorylation and nuclear localization of the A. fumigatus mitogen-activated protein kinase (MAPK) MpkA, which is involved in maintaining cell wall integrity, protection against ROS, and secondary metabolism (Jain et al., 2011). Moreover, MpkA-deficiency increases siderophore production. The TF targeted by MpkA signaling remains to be identified. Remarkably, despite its dramatic effect on in vitro growth rate, MpkA-deficiency does not affect virulence of A. fumigatus (Valiante et al., 2008).

Similar to iron, zinc plays a critical role in a diverse array of biochemical processes, but excess of zinc is deleterious. Consequently, regulation of zinc homeostasis by the TF ZafA is essential for virulence of $A$. fumigatus. Iron starvation causes zinc hypersensitive and therefore iron depletion changes cellular zinc handling by downregulating zinc uptake and upregulation of vacuolar zinc detoxification (Yasmin et al., 2009). HapX appears to play a critical role in coordination of zinc and iron homeostasis as its deficiency causes zinc hypersensitivity during iron starvation. These data demonstrate the importance of cellular metal balancing.

\section{CONCLUSION AND PERSPECTIVES}

The understanding of the role of iron in fungal pathogenicity has advanced enormously in recent years. Together with the transcriptional upregulation of the high-affinity iron acquisition systems during initiation of murine infection (McDonagh et al., 2008), the attenuated virulence caused by defects in SB or HapX confirms that A. fumigatus faces iron limitation during mammalian infection. Thus, human protection against $A$. fumigatus includes growth inhibition by polymorphonuclear leukocytes via lactoferrin-mediated iron depletion and possibly siderocalinmediated scavenging of siderophores (Fluckinger et al., 2004; Zarember et al., 2007). On the other hand, increased bone marrow iron stores represent an independent risk factor for invasive aspergillosis (Kontoyiannis et al., 2007).

The current difficulties in diagnosis and treatment of aspergillosis are reflected by the high mortality rate of this infectious disease (Tekaia and Latge, 2005). The essentiality of iron and the differences in iron handling between mammals and fungi 
like Aspergilli might help to improve therapy and diagnosis of fungal infections. Specifically, the unique fungal siderophore system represents a promising target for selective therapeutic intervention. Noteworthy, SIT constitute one of few protein families that are unique to fungi (Hsiang and Baillie, 2005). Their absence in prokaryotes and other eukaryotes might enable specific inhibition or drug delivery during infection by a "Trojan horse" approach (Miller et al., 2009), whereby antifungal agents are covalently attached to siderophores and selectively imported by fungi.

Moreover, the enzymatic and regulatory links between siderophore and ergosterol biosynthetic pathways might be crucial for optimization of treatment of infections caused by siderophoreproducing fungi. The potential of iron chelation therapy is indi-

\section{REFERENCES}

Allen, G., Bromley, M., Kaye, S. J., Keszenman-Pereyra, D., Zucchi, T. D., Price, J., Birch, M., Oliver, J. D., and Turner, G. (2011). Functional analysis of a mitochondrial phosphopantetheinyl transferase (PPTase) gene pptB in Aspergillus fumigatus. Fungal Genet Biol. 48, 456-464.

Almeida, R. S., Wilson, D., and Hube, B. (2009). Candida albicans iron acquisition within the host. FEMS Yeast Res. 9, 1000-1012.

Baek, Y. U., Li, M., and Davis, D. A. (2008). Candida albicans ferric reductases are differentially regulated in response to distinct forms of iron limitation by the Rim101 and CBF transcription factors. Eukaryotic Cell 7, 1168-1179.

Ben-Ami, R., Lewis, R. E., Leventakos, K., Latge, J. P., and Kontoyiannis, D. P. (2010). Cutaneous model of invasive aspergillosis. Antimicrob. Agents Chemother. 54, 1848-1854.

Bien, C. M., and Espenshade, P. J. (2010). Sterol regulatory element binding proteins in fungi: hypoxic transcription factors linked to pathogenesis. Eukaryotic Cell 9, 352-359.

Blatzer, M., Barker, B. M., Willger, S. D., Beckmann, N., Blosser, S. J., Cornish, E. J., Mazurie, A., Grahl, N., Haas, H., and Cramer, R. A. (2011a). SREBP coordinates iron and ergosterol homeostasis to mediate triazole drug and hypoxia responses in the human fungal pathogen Aspergillus fumigatus. PLoS Genet. 7, e1002374. doi:10.1371/journal.pgen.1002374

Blatzer, M., Binder, U., and Haas, H. (2011b). The metalloreductase FreB is involved in adaptation of Aspergillus fumigatus to iron starvation. Fungal Genet. Biol. 48, 1027-1033.

Blatzer, M., Schrettl, M., Sarg, B., Lindner, H. H., Pfaller, K., and Haas,
H. (2011c). SidL, an Aspergillus fumigatus transacetylase involved in biosynthesis of the siderophores ferricrocin and hydroxyferricrocin. Appl. Environ. Microbiol. 77, 4959-4966.

Chamilos, G., Bignell, E. M., Schrettl, M., Lewis, R. E., Leventakos, K., May, G. S., Haas, H., and Kontoyiannis, D. P. (2010). Exploring the concordance of Aspergillus fumigatus pathogenicity in mice and Toll-deficient flies. Med. Mycol. 48, 506-510.

Chang, Y. C., Bien, C. M., Lee, H., Espenshade, P. J., and Kwon-Chung, K. J. (2007). Srelp, a regulator of oxygen sensing and sterol homeostasis, is required for virulence in Cryptococcus neoformans. Mol. Microbiol. 64, 614-629.

Chen, C., Pande, K., French, S. D., Tuch, B. B., and Noble, S. M. (2011). An iron homeostasis regulatory circuit with reciprocal roles in Candida albicans commensalism and pathogenesis. Cell Host Microbe 10, 118-135.

Chun, C. D., Liu, O. W., and Madhani, H. D. (2007). A link between virulence and homeostatic responses to hypoxia during infection by the human fungal pathogen Cryptococcus neoformans. PLoS Pathog. 3, e22. doi:10.1371/journal.ppat.0030022

Eichhorn, H., Lessing, F., Winterberg, B., Schirawski, J., Kamper, J., Muller, P., and Kahmann, R. (2006). A ferroxidation/permeation iron uptake system is required for virulence in Ustilago maydis. Plant Cell 18, 3332-3345.

Eisendle, M., Oberegger, H., Buttinger, R., Illmer, P., and Haas, H. (2004). Biosynthesis and uptake of siderophores is controlled by the PacC-mediated ambient-pH Regulatory system in Aspergillus nidulans. Eukaryotic Cell 3, 561-563. Eisendle, M., Oberegger, H., Zadra, I., and Haas, H. (2003). The siderophore system is essential

cated by the synergistic effect of iron chelators and antifungal drugs demonstrated in vitro and in a murine aspergillosis model (Zarember et al., 2009; Ibrahim et al., 2010). Moreover, the recently demonstrated imaging of invasive pulmonary aspergillosis in a rat model, based on positron emission tomography (PET)-visualized fungal accumulation of TAFC-chelated ${ }^{68} \mathrm{Gallium}$, emphasizes the potential of siderophores in diagnosis of fungal infections (Petrik et al., 2010a,b).

\section{ACKNOWLEDGMENTS}

Work in the authors' laboratory is supported by Austrian Science Foundation Grants FWF P-21643-B11 and I-282-B09. Moreover, support by the European Science Foundation via its Research Networking Programme FUMINOMICS is acknowledged.

for viability of Aspergillus nidulans: functional analysis of two genes encoding l-ornithine $\mathrm{N}$ 5-monooxygenase (sidA) and a non-ribosomal peptide synthetase (sidC). Mol. Microbiol. 49, 359-375.

Eisendle, M., Schrettl, M., Kragl, C., Muller, D., Illmer, P., and Haas, H. (2006). The intracellular siderophore ferricrocin is involved in iron storage, oxidative-stress resistance, germination, and sexual development in Aspergillus nidulans. Eukaryotic Cell 5, 1596-1603.

Fluckinger, M., Haas, H., Merschak, P., Glasgow, B. J., and Redl, B. (2004). Human tear lipocalin exhibits antimicrobial activity by scavenging microbial siderophores. Antimicrob. Agents Chemother. 48, 3367-3372.

Frederick, R. E., Mayfield, J. A., and Dubois, J. L. (2011). Regulated $\mathrm{O} 2$ activation in flavin-dependent monooxygenases. J. Am. Chem. Soc. $133,12338-12341$.

Ganz, T. (2009). Iron in innate immunity: starve the invaders. Curr. Opin. Immunol. 21, 63-67.

Greenshields, D. L., Liu, G., Feng, J., Selvaraj, G., and Wei, Y. (2007). The siderophore biosynthetic gene SID1, but not the ferroxidase gene FET3, is required for full Fusarium graminearum virulence. Mol. Plant Pathol. $8,411-421$.

Haas, H., Eisendle, M., and Turgeon, B. G. (2008). Siderophores in fungal physiology and virulence. Annu. Rev. Phytopathol. 46, 149-187.

Haas, H., Schoeser, M., Lesuisse, E., Ernst, J. F., Parson, W., Abt, B., Winkelmann, G., and Oberegger, H. (2003). Characterization of the Aspergillus nidulans transporters for the siderophores enterobactin and triacetylfusarinine C. Biochem. J. 371, 505-513.

Haas, H., Zadra, I., Stoffler, G., and Angermayr, K. (1999). The
Aspergillus nidulans GATA factor SREA is involved in regulation of siderophore biosynthesis and control of iron uptake. J. Biol. Chem. 274, 4613-4619.

Halliwell, B., and Gutteridge, J. M. (1984). Oxygen toxicity, oxygen radicals, transition metals and disease. Biochem. J. 219, 1-14.

Hissen, A. H., Chow, J. M., Pinto L. J., and Moore, M. M. (2004). Survival of Aspergillus fumigatus in serum involves removal of iron from transferrin: the role of siderophores. Infect. Immun. 72, 1402-1408.

Hissen, A. H., and Moore, M. M. (2005). Site-specific rate constants for iron acquisition from transferrin by the Aspergillus fumigatus siderophores $\mathrm{N}^{\prime}, \mathrm{N}^{\prime \prime}, \mathrm{N}^{\prime \prime \prime}$-triacetylfusarinine $\mathrm{C}$ and ferricrocin. J. Biol. Inorg. Chem. 10, 211-220.

Hissen, A. H., Wan, A. N., Warwas, M. L., Pinto, L. J., and Moore, M. M. (2005). The Aspergillus fumigatus siderophore biosynthetic gene sidA, encoding L-ornithine N5oxygenase, is required for virulence. Infect. Immun. 73, 5493-5503.

Horbach, R., Graf, A., Weihmann, F., Antelo, L., Mathea, S., Liermann, J. C., Opatz, T., Thines, E., Aguirre, J., and Deising, H. B. (2009). Sfp-type $4^{\prime}$-phosphopantetheinyl transferase is indispensable for fungal pathogenicity. Plant Cell 21, 3379-3396.

Hortschansky, P., Eisendle, M., AlAbdallah, Q., Schmidt, A. D., Bergmann, S., Thon, M., Kniemeyer O., Abt, B., Seeber, B., Werner, E. R., Kato, M., Brakhage, A. A., and Haas, H. (2007). Interaction of HapX with the CCAAT-binding complex - a novel mechanism of gene regulation by iron. EMBO J. 26, 3157-3168.

Hsiang, T., and Baillie, D. L. (2005). Comparison of the yeast proteome to other fungal genomes to find core fungal genes. J. Mol. Evol. 60, 475-483. 
Hsu, P. C., Yang, C. Y., and Lan, C. Y. (2011). Candida albicans Hap43 is a repressor induced under lowiron conditions and essential for iron-responsive transcriptional regulation and virulence. Eukaryotic Cell 10, 207-225.

Hughes, A. L., Todd, B. L., and Espenshade, P. J. (2005). SREBP pathway responds to sterols and functions as an oxygen sensor in fission yeast. Cell $120,831-842$.

Hwang, L. H., Mayfield, J. A., Rine, J., and Sil, A. (2008). Histoplasma requires SID1, a member of an iron-regulated siderophore gene cluster, for host colonization. PLoS Pathog. 4, e1000044. doi:10.1371/journal.ppat.1000044

Ibrahim, A. S., Gebremariam, T., French, S. W., Edwards, J. E. Jr., and Spellberg, B. (2010). The iron chelator deferasirox enhances liposomal amphotericin B efficacy in treating murine invasive pulmonary aspergillosis. J. Antimicrob. Chemother. 65, 289-292.

Jain, R., Valiante, V., Remme, N., Docimo, T., Heinekamp, T., Hertweck, C., Gershenzon, J., Haas, H., and Brakhage, A. A. (2011). The MAP kinase MpkA controls cell wall integrity, oxidative stress response, gliotoxin production and iron adaptation in Aspergillus fumigatus. Mol. Microbiol. 82, 39-53.

Jbel, M., Mercier, A., and Labbe, S. (2011). Grx4 monothiol glutaredoxin is required for iron limitation-dependent inhibition of Fep1. Eukaryotic Cell 10, 629-645.

Jung, W. H., and Kronstad, J. W. (2008). Iron and fungal pathogenesis: a case study with Cryptococcus neoformans. Cell. Microbiol. 10, 277-284.

Jung, W. H., Saikia, S., Hu, G., Wang, J., Fung, C. K., D'souza, C., White, R., and Kronstad, J. W. (2010). HapX positively and negatively regulates the transcriptional response to iron deprivation in Cryptococcus neoformans. PLoS Pathog. 6, e1001209. doi: 10.1371/journal.ppat.1001209

Jung, W. H., Sham, A., Lian, T., Singh, A., Kosman, D. J., and Kronstad, J. W. (2008). Iron source preference and regulation of iron uptake in Cryptococcus neoformans. PLoS Pathog. 4, e45. doi:10.1371/journal.ppat.0040045

Kaplan, C. D., and Kaplan, J. (2009). Iron acquisition and transcriptional regulation. Chem. Rev. 109, 4536-4552.

Kim, K. D., Kim, H. J., Lee, K. C., and Roe, J. H. (2011). Multi-domain CGFS-type glutaredoxin Grx4 regulates iron homeostasis via direct interaction with a repressor Fep1 in fission yeast. Biochem. Biophys. Res. Commun. 408, 609-614.

Kontoyiannis, D. P., Chamilos, G., Lewis, R. E., Giralt, S., Cortes, J., Raad, I. I., Manning, J. T., and Han, X. (2007). Increased bone marrow iron stores is an independent risk factor for invasive aspergillosis in patients with high-risk hematologic malignancies and recipients of allogeneic hematopoietic stem cell transplantation. Cancer 110, 1303-1306.

Kosman, D. J. (2010). Redox cycling in iron uptake, efflux, and trafficking. J. Biol. Chem. 285, 26729-26735.

Kragl, C., Schrettl, M., Abt, B., Sarg, B., Lindner, H. H., and Haas, H. (2007). EstB-mediated hydrolysis of the siderophore triacetylfusarinine $\mathrm{C}$ optimizes iron uptake of Aspergillus fumigatus. Eukaryotic Cell 6, 1278-1285.

Labbe, S., Pelletier, B., and Mercier, A. (2007). Iron homeostasis in the fission yeast Schizosaccharomyces pombe. Biometals 20, 523-537.

Lamb, T. M., Xu, W., Diamond, A., and Mitchell, A. P. (2001). Alkaline response genes of Saccharomyces cerevisiae and their relationship to the RIM101 pathway. J. Biol. Chem. 276, 1850-1856.

Liu, H., Gravelat, F. N., Chiang, L. Y., Chen, D., Vanier, G., Ejzykowicz, D. E., Ibrahim, A. S., Nierman, W. C., Sheppard, D. C., and Filler, S. G. (2010). Aspergillus fumigatus AcuM regulates both iron acquisition and gluconeogenesis. Mol. Microbiol. 78, 1038-1054.

McDonagh, A., Fedorova, N. D., Crabtree, J., Yu, Y., Kim, S., Chen, D., Loss, O., Cairns, T., Goldman, G., Armstrong-James, D., Haynes, K., Haas, H., Schrettl, M., May, G., Nierman, W. C., and Bignell, E. (2008). Sub-telomere directed gene expression during initiation of invasive aspergillosis. PLoS Pathog. 4, el000154. doi:10.1371/journal.ppat.1000154

Mei, B., Budde, A. D., and Leong, S. A. (1993). sid1, a gene initiating siderophore biosynthesis in Ustilago maydis: molecular characterization, regulation by iron, and role in phytopathogenicity. Proc. Natl. Acad. Sci. U.S.A. 90, 903-907.

Mercier, A., and Labbe, S. (2009). Both Php4 function and subcellular localization are regulated by iron via a multistep mechanism involving the glutaredoxin Grx4 and the exportin Crm1. J. Biol. Chem. 284, 20249-20262.
Miller, M. J., Zhu, H., Xu, Y., Wu, C., Walz, A. J., Vergne, A., Roosenberg, J. M., Moraski, G., Minnick, A. A., Mckee-Dolence, J., Hu, J., Fennell, K., Kurt Dolence, E., Dong, L., Franzblau, S., Malouin, F., and Mollmann, U. (2009). Utilization of microbial iron assimilation processes for the development of new antibiotics and inspiration for the design of new anticancer agents. Biometals 22, 61-75.

Nevitt, T., and Thiele, D. J. (2011). Host iron withholding demands siderophore utilization for Candida glabrata to survive macrophage killing. PLoS Pathog. 7, e1001322. doi:10.1371/journal.ppat.1001322

Nobile, C. J., Solis, N., Myers, C. L., Fay, A. J., Deneault, J. S., Nantel, A., Mitchell, A. P., and Filler, S. G. (2008). Candida albicans transcription factor Rim101 mediates pathogenic interactions through cell wall functions. Cell. Microbiol. 10, 2180-2196.

Oberegger, H., Eisendle, M., Schrettl, M., Graessle, S., and Haas, H. (2003). $\quad 4^{\prime}$-phosphopantetheinyl transferase-encoding npgA is essential for siderophore biosynthesis in Aspergillus nidulans. Curr. Genet. 44 211-215.

Oberegger, H., Schoeser, M., Zadra, I., Abt, B., and Haas, H. (2001). SREA is involved in regulation of siderophore biosynthesis, utilization and uptake in Aspergillus nidulans. Mol. Microbiol. 41, 1077-1089.

Oberegger, H., Zadra, I., Schoeser, M., Abt, B., Parson, W., and Haas, H. (2002). Identification of members of the Aspergillus nidulans SREA regulon: genes involved in siderophore biosynthesis and utilization. Biochem. Soc. Trans. 30, 781-783.

Oberegger, H., Zadra, I., Schoeser, M. and Haas, H. (2000). Iron starvation leads to increased expression of $\mathrm{Cu} / \mathrm{Zn}$-superoxide dismutase in Aspergillus. FEBS Lett. 485, 113-116. Oide, S., Moeder, W., Haas, H., Krasnoff, S., Gibson, D., Yoshioka, K., and Turgeon, B. G. (2006). NPS6, encoding a non-ribosomal peptide synthetase involved in siderophore-mediated iron metabolism, is a conserved virulence determinant of plant pathogenic ascomycetes. Plant Cell 18, 2836-2853.

O'Meara, T. R., Norton, D., Price, M. S., Hay, C., Clements, M. F., Nichols, C. B., and Alspaugh, J. A. (2010). Interaction of Cryptococcus neoformans Rim101 and protein kinase A regulates capsule. PLoS Pathog. 6, el000776. doi:10.1371/journal.ppat.1000776

Petrik, M., Haas, H., Dobrozemsky, G., Lass-Florl, C., Helbok, A., Blatzer, M., Dietrich, H., and Decristoforo, C. (2010a). 68Ga-siderophores for PET imaging of invasive pulmonary aspergillosis: proof of principle. $J$. Nucl. Med. 51, 639-645.

Petrik, M., Haas, H., Schrettl, M., Helbok, A., Blatzer, M., and Decristoforo, C. (2010b). In vitro and in vivo evaluation of selected (68) Ga-siderophores for infection imaging. Nucl. Med. Biol. doi: 10.1016/j.nucmedbio.2011.09.012. [Epub ahead of print].

Philpott, C. C., and Protchenko, O. (2008). Response to iron deprivation in Saccharomyces cerevisiae. Eukaryotic Cell 7, 20-27.

Ramanan, N., and Wang, Y. (2000). A high-affinity iron permease essential for Candida albicans virulence. Science 288, 1062-1064.

Ratledge, C., and Dover, L. G. (2000). Iron metabolism in pathogenic bacteria. Annu. Rev. Microbiol. 54, 881-941.

Schrettl, M., Beckmann, N., Varga, J. Heinekamp, T., Jacobsen, I. D., Jochl, C., Moussa, T. A., Wang, S., Gsaller, F., Blatzer, M., Werner, E. R., Niermann, W. C., Brakhage, A. A., and Haas, H. (2010a). HapX-mediated adaptation to iron starvation is crucial for virulence of Aspergillus fumigatus. PLoS Pathog. 6, e1001124. doi:10.1371/journal.ppat.1001124

Schrettl, M., Ibrahim-Granet, O., Droin, S., Huerre, M., Latge, J. P., and Haas, H. (2010b). The crucial role of the Aspergillus fumigatus siderophore system in interaction with alveolar macrophages. Microbes Infect. 12, 1035-1041.

Schrettl, M., Bignell, E., Kragl, C., Joechl, C., Rogers, T., Arst, H. N. Jr., Haynes, K., and Haas, H. (2004a). Siderophore biosynthesis but not reductive iron assimilation is essential for Aspergillus fumigatus virulence. J. Exp. Med. 200, 1213-1219.

Schrettl, M., Winkelmann, G., and Haas, H. (2004b). Ferrichrome in Schizosaccharomyces pombe - an iron transport and iron storage compound. Biometals 17, 647-654.

Schrettl, M., Bignell, E., Kragl, C. Sabiha, Y., Loss, O., Eisendle, M., Wallner, A., Arst, H. N. Haynes, K., and Haas, H. (2007). Distinct roles for intra and extracellular siderophores during Aspergillus fumigatus infection. PLoS Pathog. 3, e128. doi:10.1371/journal.ppat.0030128

Schrettl, M., and Haas, H. (2011). Iron homeostasis - Achilles' heel of 
Aspergillus fumigatus? Curr. Opin. Microbiol. 14, 400-405.

Schrettl, M., Kim, H. S., Eisendle, M., Kragl, C., Nierman, W. C., Heinekamp, T., Werner, E. R., Jacobsen, I., Illmer, P., Yi, H., Brakhage, A. A., and Haas, H. (2008). SreA-mediated iron regulation in Aspergillus fumigatus. Mol. Microbiol. 70, 27-43.

Seifert, M., Nairz, M., Schroll, A., Schrettl, M., Haas, H., and Weiss, G. (2008). Effects of the Aspergillus fumigatus siderophore systems on the regulation of macrophage immune effector pathways and iron homeostasis. Immunobiology 213, 767-778.

Slater, J. L., Gregson, L., Denning, D. W., and Warn, P. A. (2011). Pathogenicity of Aspergillus fumigatus mutants assessed in Galleria mellonella matches that in mice. Med. Mycol. 49(Suppl. 1), S107-S113.

Tekaia, F., and Latge, J. P. (2005). Aspergillus fumigatus: saprophyte or pathogen? Curr. Opin. Microbiol. 8, 385-392.

Thon, M., Al Abdallah, Q., Hortschansky, P., Scharf, D. H., Eisendle, M., Haas, H., and Brakhage, A. A. (2010). The CCAAT-binding complex coordinates the oxidative stress response in eukaryotes. Nucleic Acids Res. 38, 1098-1113.

Valiante, V., Heinekamp, T., Jain, R., Hartl, A., and Brakhage, A. A. (2008). The mitogen-activated protein kinase MpkA of Aspergillus fumigatus regulates cell wall signaling and oxidative stress response. Fungal Genet. Biol. 45, 618-627.

Vodisch, M., Albrecht, D., Lessing, F., Schmidt, A. D., Winkler, R., Guthke, R., Brakhage, A. A., and Kniemeyer, O. (2009). Two-dimensional proteome reference maps for the human pathogenic filamentous fungus Aspergillus fumigatus. Proteomics 9, 1407-1415.

Wallner, A., Blatzer, M., Schrettl, M., Sarg, B., Lindner, H., and Haas, H. (2009). Ferricrocin, a siderophore involved in intra and transcellular iron distribution in Aspergillus fumigatus. Appl. Environ. Microbiol. 75, 4194-4196.

Weinberg, E. D. (2009). Iron availability and infection. Biochim. Biophys. Acta 1790, 600-605.

Willger, S. D., Puttikamonkul, S., Kim, K. H., Burritt, J. B., Grahl, N., Metzler, L. J., Barbuch, R., Bard, M., Lawrence, C. B., and Cramer, R. A. Jr. (2008). A sterol- regulatory element binding protein is required for cell polarity, hypoxia adaptation, azole drug resistance, and virulence in Aspergillus fumigatus. PLoS Pathog. 4, e1000200. doi:10.1371/journal.ppat.1000200

Yasmin, S., Abt, B., Schrettl, M., Moussa, T. A., Werner, E. R., and Haas, H. (2009). The interplay between iron and zinc metabolism in Aspergillus fumigatus. Fungal Genet. Biol. 46, 707-713.

Yasmin, S., Alcazar-Fuoli, L., Grundlinger, M., Puempel, T., Cairns, T., Blatzer, M., Lopez, J. F. Grimalt, J. O., Bignell, E., and Haas, H. (2011). Mevalonate governs interdependency of ergosterol and siderophore biosyntheses in the fungal pathogen Aspergillus fumigatus. Proc. Natl. Acad. Sci. U.S.A. doi 10.1073/pnas.1106399108. [Epub ahead of print].

Zarember, K. A., Cruz, A. R., Huang, C. Y., and Gallin, J. I. (2009). Antifungal activities of natural and synthetic iron chelators alone and in combination with azole and polyene antibiotics against Aspergillus fumigatus. Antimicrob. Agents Chemother. 53, 2654-2656.

Zarember, K. A., Sugui, J. A., Chang, Y. C., Kwon-Chung, K. J., and Gallin, J.
I. (2007). Human polymorphonuclear leukocytes inhibit Aspergillus fumigatus conidial growth by lactoferrin-mediated iron depletion. J. Immunol. 178, 6367-6373.

Conflict of Interest Statement: The author declares that the research was conducted in the absence of any commercial or financial relationships that could be construed as a potential conflict of interest.

Received:06 December 2011; paper pending published: 03 January 2012; accepted: 16 January 2012; published online: 06 February 2012.

Citation: Haas H (2012) Iron - a key nexus in the virulence of Aspergillus fumigatus. Front. Microbio. 3:28. doi: 10.3389/fmicb.2012.00028

This article was submitted to Frontiers in Microbial Immunology, a specialty of Frontiers in Microbiology.

Copyright (c) 2012 Haas. This is an open-access article distributed under the terms of the Creative Commons Attribution Non Commercial License, which permits non-commercial use, distribution, and reproduction in other forums, provided the original authors and source are credited. 